\title{
Creating 3-dimensional molecular models to help students visualize stereoselective reaction pathways.
}

Matthew O’Brien*

School of Physical and Geographical Sciences, Keele University, Keele, Borough of

Newcastle-under-Lyme, Staffordshire, ST5 5BG, UK.

\section{ABSTRACT}

An approach to the creation of learning materials which aid the visualization of stereoselective reaction pathways is presented. Molecular editing software can be used

10 to create models of various transition-state geometries. These 3-dimensional models can be manipulated, using suitable visualization software, to select relevant viewpoints. By using an overlay annotation tool, line-diagrams can be drawn directly over these 3dimensional representations. This may help students to make representational translations between the 3-dimensional structures (transition-states etc) and the 2-

15 dimensional diagrams typically used to depict these objects.

GRAPHICAL ABSTRACT
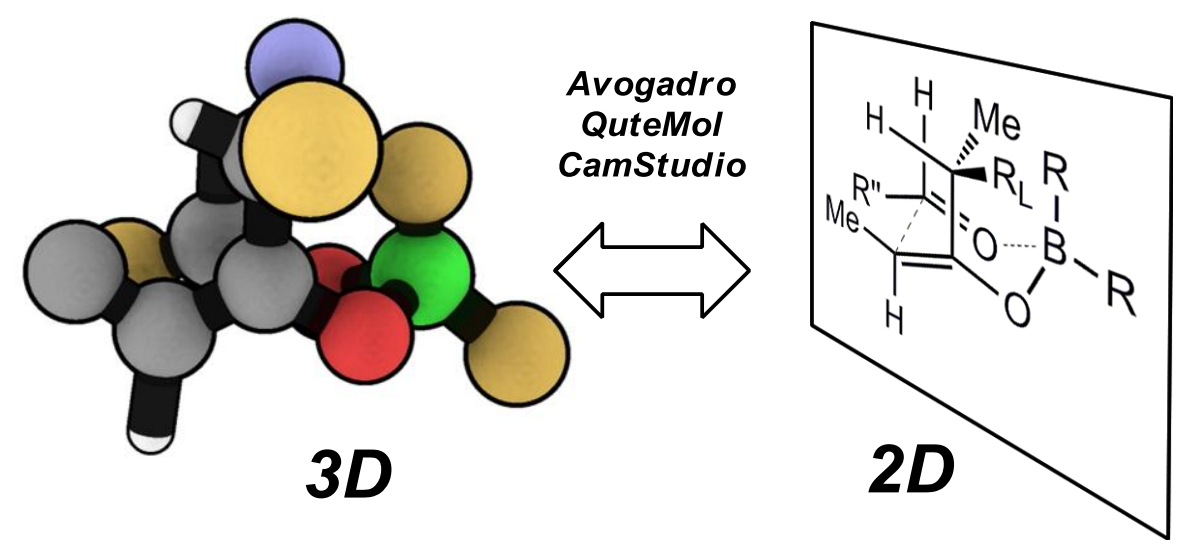

KEYWORDS 
Upper-Division Undergraduate, Organic Chemistry, Multimedia-Based Learning, Stereochemistry.

\section{EXAMPLE OF COURSE MATERIAL}

As an illustrative example of a challenging stereoselective reaction pathway, a particular slide from one of our $3^{\text {rd }}$ year optional B.Sc. courses - 'Diastereoselectivity in diagram of the proposed 'Zimmerman-Traxler' transition-state ${ }^{5}$ for a boron mediated aldol coupling of an $\alpha$-chiral ethyl ketone with an aldehyde via the $E$-enolate. The 
rationale for this transition-state is based on: 1) Adoption of a 'chair' conformation for the 6-membered transition-state. 2) Adoption of an enolate conformation which minimizes $\mathrm{A}_{1,3}$ strain between the $E$-methyl group and the substituent on the $\alpha$-carbon. This places the smallest of the $\alpha$-substituents (hydrogen) in the plane of the enolate double bond. 3) Approach by the aldehyde towards the least hindered 'face' of the enolate, i.e. closer to the methyl group rather than the large alkyl group $\mathrm{R}_{\mathrm{L}}$. In other words, $\mathrm{R}_{\mathrm{L}}$ is on the 'outside' of the transition-state, in a sterically less demanding position. 6

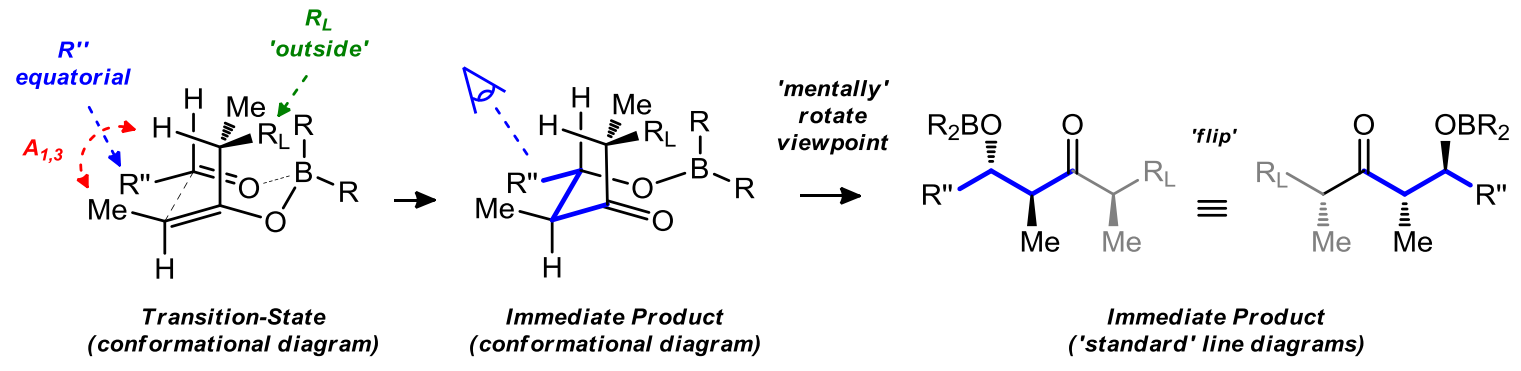

Figure 1: Course notes slide for the aldol reaction of $E$-boron-enolates of $\alpha$-chiral ketones via a Zimmerman-Traxler type transition-state. The Immediate Product is drawn in the same conformation as the transition-state. The bonds highlighted in blue in the Immediate Product conformational diagram indicate which bonds will be 'in the plane of the paper' of the 'standard' line diagram on the right. The required viewpoint for this is indicated with the eye symbol. The stereochemical configuration in the 'greyed out' section of the product line diagrams is already known from the starting materials.

There are several aspects of this example which highlight various areas of difficulty students have with visualization. Firstly, whilst some students have the ability to form a 3-dimensional mental representation of the transition-state from the conformational diagram used to depict it, others find this quite difficult. Perhaps more difficult is the 'mental' rotation of the viewpoint required to go from the conformational diagram of the Immediate Product to the 'standard' line diagram, even when the required viewpoint 
and the bonds which are 'in the plane of the paper' in the new viewpoint are indicated. Finally, flipping the final product either horizontally or vertically can also present difficulty.

\section{APPROACH TO VISUALIZATION}

By using 3-dimensional models with a combination of screen-casting and 'virtualtransparency' applications, it is possible to clearly demonstrate the relationships between starting-materials, transition-state geometries and products (and their 3dimensional and 2-dimensional depictions) in a straightforward manner.

A combination of real (e.g. Molymod) and virtual molecular models were used. Real models have the obvious advantage that students can physically interact with them and actually see them in 3-dimensions. An advantage of using virtual models is that bondlengths, bond-angles and valencies are not restricted in any way. In addition, virtual models are essentially free, assuming free access to a computer, and take no time to reconstruct, thus allowing students to easily swap between several models. package was used for their creation. Importantly, Avogadro allows the user to build 3dimensional molecules from scratch and also permits the arbitrary placement and movement of different atoms, regardless of bond-length or bond-angles. Individual atoms or groups of atoms can be selected and dragged into the desired positions. As 95 such, it is reasonably straightforward to construct any particular arrangement required. In addition, certain structural motifs, for instance the chair conformation of a 6- 
membered ring, can also be reached very easily using the built-in energy minimization routines. These can then serve as a convenient starting point for other structures, e.g. Zimmerman-Traxler transition-states. Another important feature of Avogadro is the ability to select any bond and rotate around the dihedral angle arbitrarily. This is very useful when creating Felkin-Anh type or antiperiplanar transition-states. In general, for a particular reaction, Avogadro was used to create a 3-dimensional model of the proposed transition-state as well as the corresponding 3-dimensional model of the product, where the atoms were arranged with the same geometry as the transitionstate. In order to facilitate clear visualization, elements other than those actually present were substituted in to represent various groups, based on their size and color. The structures of the transition-state and product geometries only differed significantly in the arrangement of bonds whilst the atoms remained more-or-less in the same position (see Supporting Information for examples of .pdb files of structures created in Avogadro).

In addition to being extremely useful in building and editing 3-dimensional structures, Avogadro is also able to display the structures with reasonable clarity. However, for structural display, the QuteMol${ }^{8}$ package, which is also freely available and open-source, was preferred. QuteMol enables high quality real-time manipulation and rendering of chemical structures. Its settings are highly configurable and a wide variety of molecular display types are available, including ball-and-stick types closely resembling the Molymod structures. It also has some built-in settings which serve as convenient start points for further adjustment. One limitation of QuteMol is that it represents all bonds as single bonds. This obviously needs to be explained when these models are being used. Also, bonds seem to be constructed in QuteMol according to the distance between atoms in the .pdb representation, so occasionally atoms need to be moved slightly further apart (in Avogadro) to avoid having bonds being inadvertently 
inserted between atoms simply because they are too close to each other. Despite these minor issues, the increased clarity and visual appeal of QuteMol made it the preferred display method. The settings used (see Supporting Information for details) allowed bold 'cartoonish' ball-and-stick structures to be displayed. Importantly, QuteMol is able to draw borders around structural features, making it easier to see which atoms/bonds are in front of other atoms/bonds.

To make connections between various views of the 3-dimensional structures and their 2-dimensional depictions, for example standard molecular line drawings, it was desirable to be able to draw line diagrams directly on top of the corresponding 3dimensional structure, and then to show only the line drawing itself. This required a 'virtual transparency' application. For this purpose, transparency tools, such as EpicPen ${ }^{9}$ or Annotate!Pro ${ }^{10}$ can be used. This creates a transparent drawing canvas on top of the entire desktop. The pen settings (e.g. colour, thickness) are configurable and the drawings can easily be turned on or off, or erased. A pen input tablet was used to draw these line diagrams (Wacom Bamboo).

A typical work-flow is shown in Figure 2. Structures created in Avogadro are opened in QuteMol, where they can be manipulated to show different viewpoints. The annotation tool, e.g. EpicPen or Annotate!Pro, is then used to draw line-diagrams over the various 3-dimensional views. The 3-dimensional view can then be temporarily removed simply by bringing any clear window to the front of the display, leaving the line diagram on its own. The line diagram can then be erased and the molecule manipulated to show another viewpoint, onto which a new line diagram can be drawn. In this way, the instructor can demonstrate the link between the various viewpoints of a particular 3dimensional representation and the corresponding 2-dimensional line diagrams. In 
addition to use in lectures, instructional videos can easily be created using screencasting software alongside this approach. CamStudio, ${ }^{11}$ a free and open-source screencasting application was used for this purpose (several videos outlining the approach are provided in the Supporting Information). By making the 3-dimensional structure files for transition-state geometries available to download on our intranet, students are also able to investigate them in their own time.
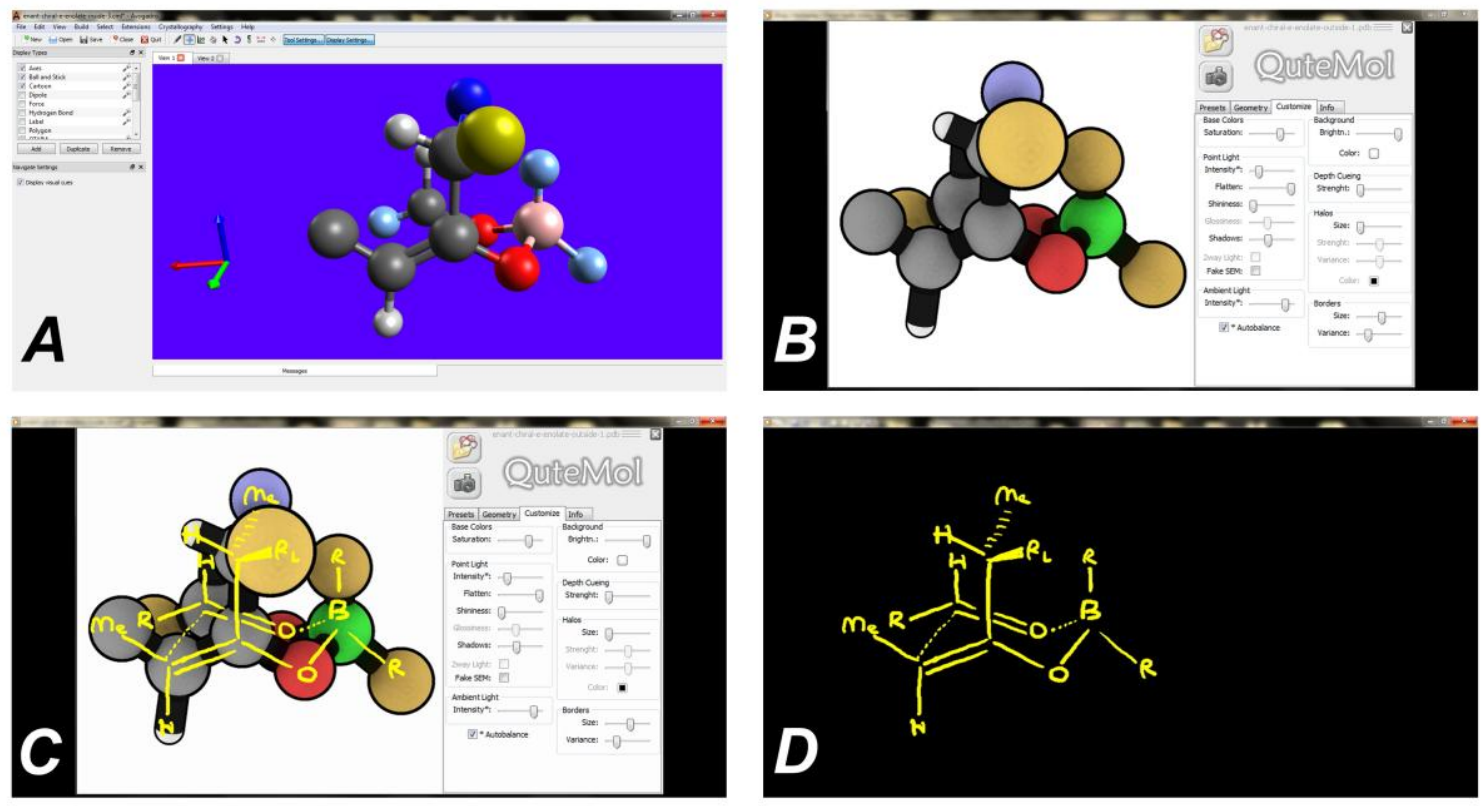

Figure 2: Workflow for stereochemical visualization. A) Transition-state geometry created in Avogadro. B) QuteMol used for manipulation and visualization, in lectures or screencasts. C) EpicPen or Annotate!Pro can be used to draw overlay line-diagram on the structure in QuteMol. D) The line diagram can be temporarily isolated by opening a blank window over QuteMol.

In addition to using virtual 3-dimensional models, real models (e.g. Molymod) can also be used with this approach (Figure 3), thereby supplementing the students' own use of physical models. To a certain extent the use of real models with this approach is more 
difficult, in that the molecules need to be held in place by hand. This means that overlay drawing cannot be done at the same time and necessitates the recording of a video of the manipulation first, then creating a new one with the annotation added. This could be alleviated if some clamping mechanism was used to hold the model steady whilst the overlaid line diagram was being drawn.
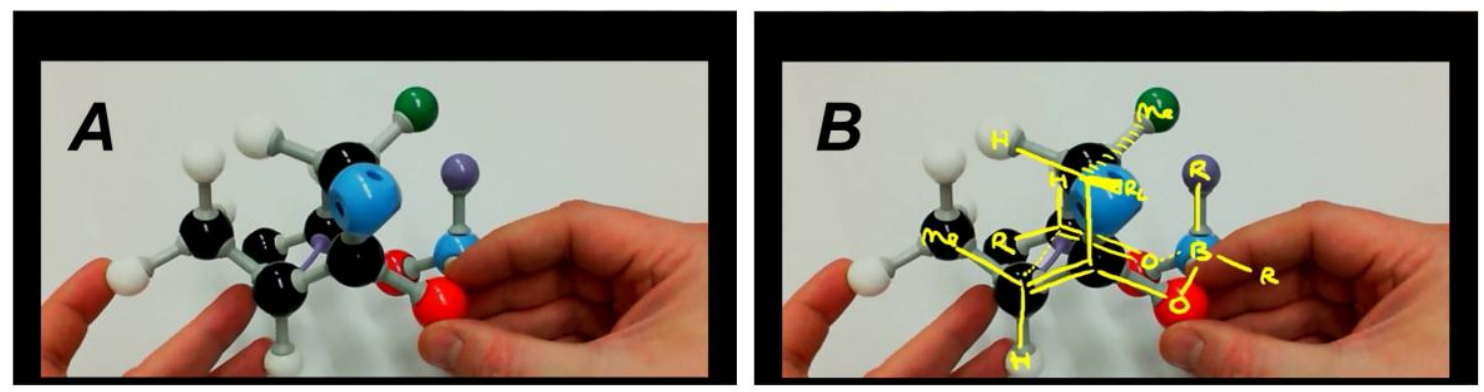

Figure 3: Use of physical models (Molymod). A) Transition-state structure made with Molymod system which is manipulated, using a webcam to make a video. B) Video played and paused to show a particular viewpoint. EpicPen or Annotate!Pro can be used to draw overlay of line diagram. CamStudio can be used to capture screen/voice to make instructional video.

\section{CONCLUSION}

Both anecdotal evidence and student feedback strongly suggest that these learning materials have been extremely beneficial to our undergraduates, especially those who previously tended to struggle with the visualization and mental manipulation of 3dimensional conformational structures. Particular areas of the course where this approach has been used include: Zimmerman-Traxler transition-states (e.g. stereoselective aldol reactions), stereoselective reductions (e.g. Evans-Saksena, Prasad) and Diels-Alder cycloadditions. These are all currently taught in the $3^{\text {rd }}$ year of our undergraduate degrees on both the B.Sc. and MChem integrated Masters courses. 
However, the approach should also find use in many other areas of organic chemistry, particularly those involving stereochemistry or conformational analysis.

ASSOCIATED CONTENT

Supporting Information

Several videos demonstrating the approach are included in the Supporting

Information, along with .pdb and .cml structure files of the Zimmerman-Traxler

transition-state and product used in the videos (QuteMol can be used to open the .pdb

file and Avogadro can be used to open the .cml file).

\section{ACKNOWLEDGEMENTS}

The author acknowledges the staff and students, past and present, of Keele University.

\section{AUTHOR INFORMATION}

200 Corresponding Author

*E-mail: m.obrien@keele.ac.uk

1. (a) Barta, N. S.; Stille, J. R. Grasping the Concepts of Stereochemistry. J. Chem. Educ. 1994, 71 (1), 20-23; (b) Gawley, R. E. Chirality Made Simple: A 1- and 2Dimensional Introduction to Stereochemistry. J. Chem. Educ. 2005, 82 (7), 10091012; (c) Lewis, D. E. The Protocenter Concept: A Method for Teaching Stereochemistry. J. Chem. Educ. 2010, 87 (6), 604-607.

2. (a) Coleman, S. L.; Gotch, A. J. Spatial Perception Skills of Chemistry Students. J. Chem. Educ. 1998, 75 (2), 206-209; (b) Barke, H.-D. Chemical education and spatial ability. J. Chem. Educ. 1993, 70 (12), 968-971; (c) Stieff, M.; Ryu, M.; Dixon, B.; Hegarty, M. The Role of Spatial Ability and Strategy Preference for Spatial Problem Solving in Organic Chemistry. J. Chem. Educ. 2012, 89 (7), 854859.

$2153 . \quad$ Talanquer, V. Threshold Concepts in Chemistry: The Critical Role of Implicit Schemas. J. Chem. Educ. 2015, 92 (1), 3-9.

4. (a) Abraham, M.; Varghese, V.; Tang, H. Using Molecular Representations To Aid Student Understanding of Stereochemical Concepts. J. Chem. Educ. 2010, 87 
(12), 1425-1429; (b) Cody, J. A.; Craig, P. A.; Loudermilk, A. D.; Yacci, P. M.;

Frisco, S. L.; Milillo, J. R. Design and Implementation of a Self-Directed

Stereochemistry Lesson Using Embedded Virtual Three-Dimensional Images in a

Portable Document Format. J. Chem. Educ. 2012, 89 (1), 29-33; (c) McCollum, B.

M.; Regier, L.; Leong, J.; Simpson, S.; Sterner, S. The Effects of Using Touch-

Screen Devices on Students' Molecular Visualization and Representational

Competence Skills. J. Chem. Educ. 2014, 91 (11), 1810-1817; (d) Springer, M. T.

Improving Students' Understanding of Molecular Structure through Broad-Based Use of Computer Models in the Undergraduate Organic Chemistry Lecture. $J$.

Chem. Educ. 2014, 91 (8), 1162-1168; (e) Francoeur, E. The Forgotten Tool: The

Design and Use of Molecular Models. Soc. Stud. Sci. 1997, 27 (1), 7-40; (f) Gupta, H. O.; Parkash, B. A Novel Multipurpose Model Set for Teaching General Chemistry. J. Chem. Educ. 1999, 76 (2), 204-205.

5. Zimmerman, H. E.; Traxler, M. D. The Stereochemistry of the Ivanov and Reformatsky Reactions. I. J. Am. Chem. Soc. 1957, 79 (8), 1920-1923.

6. Evans, D. A.; Ng, H. P.; Clark, J. S.; Rieger, D. L. Diastereoselective Anti Aldol Reactions of Chiral Ethyl Ketones. Enantioselective Processes for the Synthesis of Polypropionate Natural Products. Tetrahedron 1992, 48 (11), 2127-2142.

7. (a) Hanwell, M. D.; Curtis, D. E.; Lonie, D. C.; Vandermeersch, T.; Zurek, E.; Hutchison, G. R. Avogadro: an advanced semantic chemical editor, visualization, and analysis platform. J. Cheminf. 2012, 4 (1), 1-17; (b) "Avogadro - Free crossplatform molecule editor"'; http://avogadro.cc/ (accessed July 2016)

8. (a) Tarini, M.; Cignoni, P.; Montani, C. Ambient occlusion and edge cueing to enhance real time molecular visualization. IEEE Trans. Vis. Comput. Graph. 2006, 12 (5), 1237-1244; (b) "QuteMol"; http://qutemol.sourceforge.net/ (accessed July 2016)

9. "Epic Pen download | SourceForge.net"; https://sourceforge.net/projects/epicpen/ (accessed July 2016)

10. "Annotate!Pro | Useful for Effective Meetings, Presentations, Training, Sales, Consultants, Documentation"; http://annotatepro.com/ (accessed July 2016)

11. "CamStudio - Free Screen Recording Software"; http://camstudio.org/ (accessed July 2016) 